\title{
X-ray high-resolution diffraction using refractive lenses
}

\author{
Michael Drakopoulos \\ Diamond Light Source Ltd., Chilton, Oxfordshire OX11 OQX, United Kingdom
}

Anatoly Snigirev and Irina Snigireva

European Synchrotron Radiation Facility, 38043 Grenoble, France

Jörg Schilling

California Institute of Technology, Pasadena, California 91125

(Received 21 July 2004; accepted 1 November 2004; published online 23 December 2004)

\begin{abstract}
Refractive x-ray lenses have recently been applied for imaging and scanning microscopy with hard $\mathrm{x}$ rays. We report the application of refractive lenses in an optical scheme for high-resolution $\mathrm{x}$-ray diffraction, performed at a high brilliance synchrotron radiation source. An experimental proof of principle and a theoretical discussion are presented. In particular, we observe the x-ray diffraction pattern from a two-dimensional photonic crystal with $4.2 \mu \mathrm{m}$ periodicity, which normally is employed to scatter light in the infrared. (C) 2005 American Institute of Physics.
\end{abstract}

[DOI: $10.1063 / 1.1843282]$

Compound refractive lenses (CRL) for $\mathrm{x}$ rays ${ }^{1}$ are unique devices for hard $\mathrm{x}$-ray microscopy. By creating a microscopically small $\mathrm{x}$-ray beam, a sample can be analyzed with high spatial resolution in a scanning microscopy manner. By this, they facilitate microdiffraction, microfluorescence, and microspectroscopy, and reveal two- or threedimensional chemical and structural material properties on a microscopic scale. ${ }^{2-7}$ As well, full field imaging is possible, when $\mathrm{x}$-ray light which is diffracted from an object is imaged onto an X-ray detector by means of CRLs. ${ }^{8}$

In analogy to visible light lenses, $\mathrm{x}$-ray refractive lenses introduce a phase shift to the electromagnetic waves traveling through the lensmaterial. Knowing the refractive index and the shape of a CRL, the optical characteristics can be derived in equivalence to the known laws of visible light optics. $^{9}$

In our letter we describe the use of CRLs as optical elements for high resolution x-ray diffraction. We use the fact that a lens acts as an angular analyzer that may create a diffraction pattern of an object near its back focal plane ${ }^{10}$ that can easily be recorded with a two-dimensional detector.

We verified this concept with a diffraction experiment at the undulator beamline ID18F of the European Synchrotron Radiation Facility (ESRF). The setup is schematically shown in Fig. 1. The essential quantity for the diffraction scheme is the small source located at a large distance $r$ from the sample. From the undulator device we obtain an effective vertical source size $\sigma$ of $60 \mu \mathrm{m}^{11}$ and a horizontal source size of $800 \mu \mathrm{m}$, which we further reduce to $50 \mu \mathrm{m}$ at a distance of $32 \mathrm{~m}$ by means of horizontal slits. From the location in the experimental hutch at $r=58 \mathrm{~m}$, the effective source appears inside a solid angle $\omega=\sigma / r$ (the angular source size) of $1.9 \mu \mathrm{rad}$ horizontally and $1 \mu \mathrm{rad}$ vertically.

A wavelength $\lambda$ of $0.443 \AA$ was selected by a double crystal monochromator, with a relative bandwidth of 1.4 $\times 10^{-4}$. We place a parabolic CRL ${ }^{12}$ into the beam path, centered on the optical axis, and behind, at a distance $s$ $=1314 \mathrm{~mm}$ a high-resolution $\mathrm{x}$-ray camera. The CRL images the source in demagnification onto the image plane, and $r$ and $s$ are the corresponding object and image distances. The $\mathrm{x}$-ray camera is a CCD-based two-dimensional detector with a spatial resolution of $2 \mu \mathrm{m}$ (line spread function). ${ }^{13}$ The CRL is fabricated of $N$ individual lenses $(N=112)$ stacked behind each other on a common axis. An individual lens is made of a polycrystalline $\mathrm{Al}$ sheet into which two paraboloids are pressed from both sides. The radius of curvature at the apex of each parabola is $R=200 \mu \mathrm{m}$. The increasing absorption of $\mathrm{x}$ rays towards the outer torus of the lens limits its aperture to an effective size of $\sim 200 \mu \mathrm{m}$ in diameter (FWHM). As a test sample, we use a two-dimensional photonic crystal. ${ }^{14}$ It consists of a hexagonal whole pattern, which is fabricated into a $150 \mu \mathrm{m}$ thick Si wafer [Fig. 2(a)]. The two vectors describing the hexagonal grid, $a$ and $b$, have a length of $4.2 \mu \mathrm{m}$, and an included angle of $120^{\circ}$. The holes are almost quadratic in shape and have a size of around $2 \mu \mathrm{m}$. The photonic crystal forms a two-dimensional lattice, and the resulting lattice lines $d_{01}$ and $d_{11}$, expressed with Miller indices, have a distance of 3.6 and $2.1 \mu \mathrm{m}$, respectively. This photonic crystal is placed immediately after the lens (Fig. 1, plane $A^{\prime}$ ). At the image plane, we record the two-dimensional x-ray diffraction pattern of the crystal [Fig. 2(b)]. The successive orders of the $\{01\}$-family and $\{11\}$ family reflections are observable with the orientation according to the lattice spacing and the symmetry of the photonic

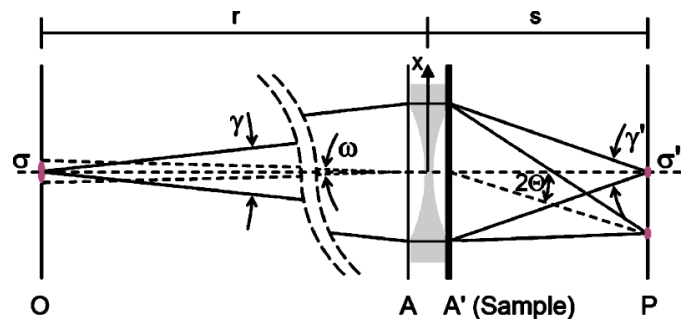

FIG. 1. Optical scheme of the diffraction instrument (dimensions not to scale). $O, A, A^{\prime}$, and $P$ are the source plane, the lens entrance and exit plane, and the detector plane, respectively. The sample is placed at $A^{\prime}$. Real and demagnified source size are indicated by $\sigma$ and $\sigma^{\prime}$. The angular source size and divergence angles before and after the lens are $\omega, \gamma$, and $\gamma^{\prime}$, respectively. $2 \Theta$ is the diffraction angle. The distance of a lens element to the optical axis is $x . r$, and $s$ are the object and the image distance of the source. (A double crystal monochromator between source and sample for selecting a particular wavelength is not displayed). 


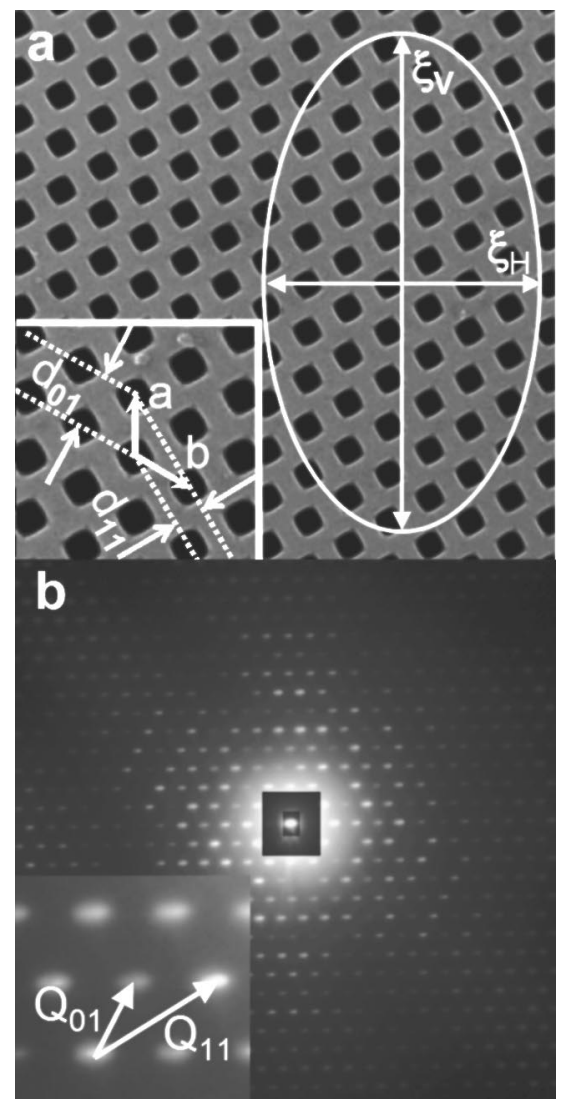

FIG. 2. (a) Scanning electron microscope image of the photonic crystal (Ref. 14) used as test sample. The inset shows the geometrical parameters relevant for diffraction. The vectors a and $b$ have a length of $4.2 \mu \mathrm{m}$. The outlined zone corresponds to the coherently illuminated sample area defined by the horizontal and vertical coherence lengths $\xi_{H}$ and $\xi_{V}$; (b) x-ray diffraction pattern recorded at an $\mathrm{x}$-ray energy of $28 \mathrm{keV}$ and a detector distance of $1314 \mathrm{~mm}$. Data are presented without any treatment. Intensities are displayed in a logarithmic gray scale to account for large dynamic range. In addition, the scaling is adapted to the higher intensities in the central regions. The first order reflections are strongly dampened by the envelope slit scattering function, which has a minimum close-by. The inset shows the reciprocal lattice vectors for the two principal reflections.

crystal. The diffraction angles are multiples of 12.3 and $21.0 \mu \mathrm{rad}$ for the $\{01\}$-reflections and the $\{11\}$-reflection, respectively. These values agree with the calculated diffraction angles $2 \Theta=\lambda / d$.

In reciprocal space, the corresponding lattice vectors $g_{01}$ and $g_{11}$ we are able to resolve have the lengths $1.75 \times 10^{-3}$ and $2.99 \times 10^{-3} \mathrm{~nm}^{-1}$, respectively. We may have a closer look at the resolution in reciprocal space that can be achieved with this method. Given the wave vectors of the incident and the scattered radiation, $k_{0}$ and $k$, the momentum transfer $q$ is defined by $q=k-k_{0}$. The perpendicular momentum transfer resolution $\Delta q_{\perp}$ of this kind of diffraction instrument can be derived in terms of resolvable detail at the detector plane. It is given by the fact that only diffraction angles $2 \Theta$ larger then the angular size of the demagnified source $\sigma^{\prime}$ can be resolved. This leads to

$$
\Delta q_{\perp}=\left|k_{0}\right| \cdot \omega,
$$

where $\left|k_{0}\right|=2 \pi / \lambda$. At a given x-ray energy, the momentum resolution depends on the angular source size and is independent of the divergence angles $\gamma$ (or $\gamma^{\prime}$ ). In our experiment, the beam illuminating the sample has an angular spread $\gamma^{\prime}$ of Downloaded 14 Dec 2005 to 131.215.225.171. Redistribution subje around $150 \mu \mathrm{rad}$, which is large compared to the diffraction angles.

The momentum transfer resolution also reflects the largest correlation length between scattering centers to give rise to interference. This correlation length is ultimately set by the transverse coherence length $\xi$ of the incoming x-ray beam, which after free propagation from the source to point at distance $r$ has the value ${ }^{15} \xi=\lambda \cdot r / \sigma=\lambda / \omega$. From here we derive the same result for the momentum resolution as in Eq. (1). The transverse coherence lengths for our particular experiment are $23 \mu \mathrm{m}$ horizontally and $44 \mu \mathrm{m}$ vertically [Fig. 2(a)].

Nearly similar momentum resolution in forward scattering may be achieved at small angle x-ray scattering (SAXS) instruments at high brilliance synchrotron radiation sources. ${ }^{16-18}$ Usually, the incoming beam divergence is kept small, in order to minimize the instrumental resolution function $R(q)$, as the scattered intensity $I(q)$ is obtained by convoluting the cross section of the sample with $R(q): I(q)$ $\propto \int S\left(q^{\prime}\right) R\left(q-q^{\prime}\right) d q^{\prime}$. Often additional collimation and angular analysis is applied by means of single crystal optics. ${ }^{19}$ These methods, however, do not account for the transverse coherence attained in the incoming beam, as was already pointed out by Sinha et al. ${ }^{20}$

Instead, we follow the standard Fresnel-Kirchhoff diffraction theory for paraxial optics, ${ }^{10}$ for the particular case of our experimental instrument (Fig. 1). The amplitude at the detector plane $P$ may be described by:

$$
\begin{aligned}
U\left(\Theta_{x}, \Theta_{y}\right)= & C \cdot \iint_{\text {lens }} \Psi_{\text {lens }}(x, y) \cdot \Psi_{\text {object }}(x, y) \\
& \times \exp [i k \cdot v(x, y)] d x d y,
\end{aligned}
$$

with

$$
v(x, y)=\frac{x^{2}+y^{2}}{2 r}+\frac{x^{2}+y^{2}}{2 s}-2 \Theta_{x} \cdot x-2 \Theta_{y} \cdot y
$$

$\Psi_{\text {lens }}$ and $\Psi_{\text {object }}$ are complex transmission functions of the lens and the sample, respectively. The phase shift $\Delta \varphi$ induced by the CRL and the corresponding transmission function are known:

$$
\Psi_{\text {lens }}=\exp (-i \Delta \varphi), \quad \Delta \varphi=\frac{2 \pi}{\lambda} \cdot \delta \cdot \frac{N \cdot\left(x^{2}+y^{2}\right)}{R},
$$

expressed with the Cartesian coordinates $x$, and $y$, centered on the optical axis in plane $A^{\prime}$ (a constant phase shift due to finite axial thickness is omitted). The quantity $\delta$ is the decrement of the refractive index (for $\mathrm{Al}$ at $28 \mathrm{keV}, \delta=6.89$ $\left.\times 10^{-7}\right)$. The focal length $f$ of the CRL is given as $1 / f$ $=1 / r+1 / s=2 N \delta / R$. From Eqs. (2) and (3) follows, that the phase-modulation induced by the lens reduces the FresnelKirchhoff integral to a Fourier transformation between the angular amplitude distribution $U\left(\Theta_{x}, \Theta_{y}\right)$ and the object transmission function $\Psi_{\text {object }}(x, y)$. The intensity distribution at the detector plane is therefore the diffraction pattern of the sample.

We introduced an $x$-ray optical system that allows to access momentum transfer resolutions in the order of $10^{-4} \mathrm{~nm}^{-1}$. Up until now, even at the current generation synchrotron sources, such a resolution has rarely been obtained, and only with additional angular collimation by means of Bonse-Hart-type crystal collimators. ${ }^{19}$ In the future, with the to AIP license or copyright, see http://apl.aip.org/apl/copyright.jsp 
straightforward fast acquisition of two-dimensional highresolution diffraction patterns with fast cameras one would be able to study static and dynamic properties within distinct volumes of the reciprocal space, with correlation lengths of up to several tens of micrometers. At present, this is possible only with visible light scattering. Moreover, the constraint to conserve the angular divergence is actually not necessary, and one may design high-resolution diffraction instruments in a compact way (present high resolution small-angle $\mathrm{x}$-ray scattering beamlines contain drift spaces of more than $10 \mathrm{~m}$ in length). Recent progress in the making of x-ray lenses allows access into the photon energy regime above $100 \mathrm{keV} .{ }^{21}$ Then the attenuation length for most media exceeds several hundred micrometers. This opens up applications in hard condensed matter and material science problems, which otherwise can be carried out with neutron radiation only. For example, a high-energy x-ray reflection scheme based on CRLs has recently been used for the study of buried interfaces. ${ }^{22,23}$

The authors would like to thank R. Rueffer, A. Chumakov, O. Leupold, and J.-P. Celse from beamline ID18 for their excellent support. As well, they appreciate Viktor Kohn's critical remarks during the preparation of the manuscript.

${ }^{1}$ A. Snigirev, V. Kohn, I. Snigireva, and B. Lengeler, Nature (London) 384, 49 (1996).

${ }^{2}$ C. G. Schroer, M. Kuhlmann, T. F. Günzler, B. Lengeler, M. Richwin, B. Griesebock, D. Lützenkirchen-Hecht, and R. Frahm, Appl. Phys. Lett. 82, 3360 (2003).

${ }^{3}$ M. Drakopoulos, J. Zegenhagen, A. Snigirev, I. Snigireva, M. Hauser, K. Eberl, V. Aristov, L. Shabel'nikov, and V. Yunkin, Appl. Phys. Lett. 81, 2279 (2002).

${ }^{4}$ S. Bohic, A. S. Simionovici, A. Snigirev, R. Ortega, G. Deves, D. Hey- mann, and C. Schroer, Appl. Phys. Lett. 78, 3544 (2001).

${ }^{5}$ C. Schroer, M. Kuhlmann, U. T. Hunger, T. F. Günzler, O. Kurapova, S. Feste, F. Frehse, B. Lengeler, M. Drakopoulos, A. Somogyi, A. S. Simionovici, A. Snigirev, I. Snigireva, C. Schug, and W. H. Schröder, Appl. Phys. Lett. 82, 1485 (2003).

${ }^{6}$ T. J. Wess, I. Alberts, J. Hiller, M. Drakopoulos, A. T. Chamberlain, and M. Collins, Calcif. Tissue Int. 70, 103 (2002).

${ }^{7}$ O. Castelnau, M. Drakopoulos, C. Schroer, I. Snigireva, A. Snigirev, and T. Ungar, Nucl. Instrum. Methods Phys. Res. A 467-468, 1245 (2001).

${ }^{8}$ B. Lengeler, C. Schroer, M. Richwin, J. Tümmler, M. Drakopoulos, A. Snigirev, and I. Snigireva, Appl. Phys. Lett. 74, 3924 (1999).

${ }^{9}$ V. Kohn, I. Snigireva, and A. Snigirev, Opt. Commun. 261, 247 (2003).

${ }^{10} \mathrm{M}$. Born and E. Wolf, Principles of Optics, 7 th ed. (Cambridge University Press, Cambridge, 1999).

${ }^{11}$ The effective source size is a convolution of the real source size and the blurring which is caused by nonideal beamline optics.

${ }^{12}$ B. Lengeler, C. Schroer, J. Tümmler, B. Benner, M. Richwin, A. Snigirev, I. Snigireva, and M. Drakopoulos, J. Synchrotron Radiat. 6, 1153 (1999).

${ }^{13}$ A. Koch, C. Raven, P. Spanne, and A. Snigirev, J. Opt. Soc. Am. A 15, 1940 (1998).

${ }^{14}$ J. Schilling, R. B. Wehrspohn, A. Birner, F. Müller, R. Hillebrand, U. Gösele, S. W. Leonard, J. P. Mondia, F. Genereux, H. M. van Driel, P. Kramper, V. Sandoghar, and K. Bush, J. Opt. A, Pure Appl. Opt. 3, S121 (2001).

${ }^{15}$ F. Zernike, Physica (Amsterdam) 5, 785 (1938).

${ }^{16}$ T. Narayanan, O. Diat, and P. Bösecke, Nucl. Instrum. Methods Phys. Res. A 467-468, 1005 (2001).

${ }^{17}$ J. Ilavsky, A. J. Allen, G. G. Long, and P. R. Jemian, Rev. Sci. Instrum. 73, 1660 (2002).

${ }^{18}$ S. Seifert, R. E. Winans, D. M. Tiede, and P. Thigarayan, J. Appl. Crystallogr. 33, 782 (2000).

${ }^{19}$ U. Bonse and M. Hart, Z. Phys. 189, 151 (1966).

${ }^{20}$ S. K. Sinha, M. Tolan and A. Gibaud, Phys. Rev. B 57, 2740 (1998).

${ }^{21}$ M. Grigoriev, L. Shabel'nikov, V. Yunkin, A. Snigirev, I. Snigireva, M. Di Michiel, S. Kuznetsov, M. Hoffmann, and E. Voges, Proc. SPIE 4501, 185 (2001).

${ }^{22}$ H. Reichert, V. Honkimäki, A. Snigirev, S. Engemann, and H. Dosch, Physica B 336, 46 (2003).

${ }^{23}$ S. Engemann, H. Reichert, H. Dosch, J. Bilgram, V. Honkimäki, and A. Snigirev, Phys. Rev. Lett. 92, 205701 (2004). 